\title{
It's the real Queen Bee. Eine Analyse des VIDEOCLIPS »NO MATTER WHAT THEY SAY« DER RAPPERIN LIL' KIM ${ }^{1}$
}

\author{
Sonja Henscher
}

Das Musikgenre Rap ist aktuell aufgrund seiner weltweiten Popularität ein viel diskutiertes Thema in den Medien und auch die Wissenschaft entwickelte in den letzten Jahren ein immer größeres Interesse an diesem vielschichtigen Phänomen. Die vielen oberflächlichen Betrachtungen haben zu einer von Vorurteilen und Klischees geprägten Diskussion geführt. Besonders zum Bereich der Inszenierungen afro-amerikanischer Interpretinnen fehlt es noch an differenzierten Erörterungen. Wenige wissenschaftliche Autorinnen und Autoren widmen sich, wie z.B. Tricia Rose in ihrer Arbeit Black Noise (1994), diesem Thema intensiver. Viele einschlägige Veröffentlichungen (z.B. Batschari 1997; Fernando 1994; Glowania/Heil 1996) gehen kaum über Basisinformationen und stereotype Betrachtungsweisen hinaus. Dies führt oft zu einer verkürzten und kolonialisierenden Darstellung.

Im Folgenden werden wesentliche Merkmale der Inszenierung der Künstlerin Lil' Kim in ihrem Clip »No Matter What They Say im Rahmen einer differenzierten Analyse unter Einbezug des spezifischen ethnischen Hintergrundes aufgezeigt. Das verwendete Analysemodell orientiert sich in erster Linie am Kriterienkatalog von Winfried Pape und Kai Thomsen (1997: 200226), der alle Ebenen eines Clips sowie die Verknüpfung von Text und Musik mit dem Bild in detaillierter Form berücksichtigt und zudem auf Produktionskriterien und zusätzliche Funktionen eingeht. An manchen Stellen wurde der Katalog erweitert, um afro-amerikanische Kommunikationsstrukturen und musikimmanente Phänomene besser beleuchten zu können. Die Bearbeitung anderer Kriterien des Modells wurde mangels Informationsmöglichkeiten (z.B. Profil des Regisseurs, Budget), Relevanz oder Resultat ausgelassen. Auf der Grundlage des Katalogs wurden Modelle zur Text-, Musik- und

1 Diese Analyse ist Teil einer umfassenderen Untersuchung (Henscher 2001), in der auch Videoclips von Lauryn Hill und Missy Elliott berücksichtigt wurden. 
vergleichenden Analyse erstellt, die zur Gewinnung der nachfolgend dargestellten Ergebnisse der Lil' Kim-Videoclipanalyse dienten.

\section{Textanalyse}

Der Text von »No Matter What They Say « ${ }^{2}$ ist geprägt von Ausdrucksformen des Black American English (BAE). ${ }^{3}$ In ihrem Videoclip geht es Lil' Kim um die bestmögliche Selbstdarstellung. Die Interpretin beschreibt sich, wie in den meisten ihrer Songs, als begehrte und reiche Frau, die um ihren Erfolg und ihre Fähigkeiten als $M C^{4}$ beneidet wird. In der ersten Strophe beschreibt sie auch ihre Freundinnen (von denen die Sängerinnen Missy Elliott und Mary J. Blige in diesem Videoclip auch im Bild erscheinen) als besonders durchsetzungsfähig, gut aussehend und erfolgreich. Zu diesem Zweck, sowie zur möglichst vorteilhaften Darstellung ihrer selbst, werden im Text hauptsächlich das Boasting ${ }^{5}$ und Jive-Begriffe ${ }^{6}$ als sprachliche Stilmittel des BAE ver-

2 Vollständiger Text unter http://ohhla.com/anonymous/lil_kim/KIM/nomatter. kim.txt (Zugriff: 16.06.2003).

3 Das BAE verstehe ich als ein afro-amerikanisches Kommunikationssystem, das verbale Sprache, Bilder- und Körpersprache umfasst. Die verbale Sprache besitzt einen regulären Sprachkörper (vgl. Buß 1998; Dauer/Hoffmann 1987ff.; Hoffmann 1994) und weist zahlreiche Dialekte auf. Sie beinhaltet neben Betonungs-, Aussprache- und grammatikalischen Regeln sowie eigenständigen Vokabularen auch spezielle sprachliche Verhaltensweisen bzw. Kommunikationsformen (Formen verbaler Manipulation, Techniken verbaler Herausforderung, z.B. Boasting, Signifying, Woofing) auf der semantischen Ebene (vgl. Buß 1998; Kochman 1972; Smitherman 1977). Diese sprachlichen Mittel sind in die Poesie afro-amerikanischer Vokalmusik, also auch in die der Rapmusik, welche der Bluespoesie verwandt ist (vgl. Hoffmann 1994; Buß 1998), eingegangen. Bei der nonverbalen, körperlichen Kommunikation ist zwischen gestischem und mimischem Verhalten zu unterscheiden, das charakteristisch für bestimmte synchron ablaufende sprachliche Verhaltensweisen ist, und dem »Silent Rap« (Buß 1998: 21), der keine Ergänzung verbaler Kommunikation darstellt, sondern für sich steht, aber die gleichen Absichten wie die sprachlichen Verhaltensweisen hat. Die Bildersprache des BAE setzt wiederum verbalsprachliche Kommunikationsformen der Manipulation und Herausforderung in visuelle Formen um.

4 »MC « ist eine im Sprachgebrauch gängige Abkürzung für »Master of Ceremony« als alternative Bezeichnung für »Rapper«.

5 Mit Boasting ist hier das Prahlen mit materiellem Wohlstand, sexuellen Qualitäten, Statussymbolen etc. gemeint. Es dient als sprachliche Verhaltensweise des BAE zur möglichst positiven Selbstdarstellung, um andere Leute für sich zu begeistern.

6 Beim Jiving im BAE wird das Gegenteil von dem gesagt, was eigentlich gemeint ist. Um die dabei übermittelten Informationen richtig zu verstehen, muss man das entsprechende Vokabular kennen, das sich ständig weiterentwickelt. Ein typischer, in Lil' Kims Text verwendeter Jive-Begriff ist z.B. »bad« (»my B-A-D- 
wendet. So betitelt P. Diddy als Gastrapper in diesem Stück Lil' Kim auch als »Queen Bee« bzw. »Queen Bitch« und meint das durchaus positiv. Das Jiving deutet hier das Schimpfwort »bitch « (Hure, Zicke) in die Bedeutung einer starken, schlauen Frau mit Durchsetzungsvermögen um. Der Begriff ist so als hohes Lob zu interpretieren, das durch den Zusatz »Queen« noch gesteigert wird. Mit dieser Umdeutung vermeidet Lil' Kim eine Einordnung in das System von >guten< und sschlechten< Frauen, die häufig in Raps von männlichen wie auch weiblichen MCs vorgenommen wird (vgl. Dufresne 1997, 302ff.). Dabei werden die »Schlechten « als oberflächlich und unmoralisch beschrieben, als Frauen, für die nur materieller Reichtum zählt und die massiv ihre weiblichen Reize einsetzen, um ihren Willen zu bekommen. Den »Guten« werden hingegen Attribute wie kultiviert, ruhig, treu, dezent gekleidet und verantwortungsbewusst zugeschrieben. Geld spielt für diese Frauen keine Rolle.

Schon auf ihrem ersten Soloalbum Hardcore (1996) bezeichnete sich Lil' Kim selbst als »Queen Bitch«. Dieser Titel ist inzwischen zu einem Markenzeichen der Rapperin geworden. Explizite sexuelle Inhalte, die teilweise aggressive Züge annehmen, sind typisch für Lil' Kims Raptexte. Der Text von »No Matter What They Say«, in dem sie lediglich ihren Sexappeal anpreist, ist in diesem Zusammenhang vergleichsweise harmlos.

Zur besseren Ausgestaltung ihres Boastings benutzt die Interpretin verschiedene Stilmittel wie Vergleich, Anapher, Synonymie ${ }^{7}$ und Zitate. Als Zitat erscheint ein Sample eines Stücks von Eric B. und Rakim (»I Know You Got Soul«, 1987), das den im Rap so wichtigen Aspekt des Styles ${ }^{8}$ der Rapperin als besonders positiv hervorhebt: "This is how it should be done, 'cause this style is identical to none. " Das direkt darauf folgende zweite Zitat aus dem Stück »Rapper's Delight« (Sugar Hill Gang, 1979) besitzt einerseits einen hohen Wiedererkennungswert, andererseits erweist Lil' Kim damit ihren

G-I-R-L-S is in the stretch $L X \ll)$, der hohe Anerkennung und Lob zum Ausdruck bringt. »Dike« (»| got a whole bunch of dikes on ninja bikes«) ist ein weiterer verwendeter Jive-Begriff, der hier »harte, starke Frau« im positiven Sinn meint, im Standard American English dagegen »Lesbe« bedeutet und dort auch als Schimpfwort eingesetzt wird.

7 Vergleiche: »Girls making faces like Ace Ventura«, »We fly like kites«, »My rocks shine like they were dipped in Cascade «. Anapher: »'Cause y'all take to my rap, 'cause my rap's the best «. Synonymie: »I'm the highest, your highest title, numero uno .

8 Der Begriff »Style « bezieht sich im Rap einerseits auf die tonale Qualität eines Raps, andererseits auf den Grad der Originalität der verbalen Vortragsweise sowie der Gesamtpräsentation der Interpretin/des Interpreten (vgl. Salaam 1996: 122). 
Respekt vor den kommerziellen Anfängen des Rap': »The hip hop, the hippie, the hippie to the hip hip hop ah ya don't stop, to rock it to the bang bang boogie, to up jump the boogie to the [boogie to] the boogedy beat.«

Wie in den meisten Rapvideoclips wurden auch in diesem Clip einzelne Wörter aus Gründen der Zensur herausgeschnitten. Bei den zensierten Vokabeln handelt es sich um den Jive-Begriff »dikes« (vgl. Anm. 5), das Wort »shit«, das hier einfach »nichts« bedeutet und die Vokabel »niggas«, die als neutrale bis positiv wertende Bezeichnung für »Männer « oder »Typen« zu interpretieren ist. Eine Zensur ist also eigentlich unbegründet und nur auf Unkenntnis oder ein Nicht-Anerkennen-Wollen des BAE zurückzuführen, was wiederum zu einem falschen Bewusstsein beim Konsumenten oder analysierenden Wissenschaftler bezüglich des afro-amerikanischen Kommunikationssystems führt.

\section{Musikalische Analyse}

Nach vielen wissenschaftlichen Ansätzen der Videoclipanalyse hat das Bild bei der Wahrnehmung von Videoclips den Vorrang (vgl. Pape/Thomsen 1997: 215). Auch dem Text wird von manchen Autoren in der Rezeption von Vokalmusik Priorität eingeräumt. Ich schließe mich jedoch der Meinung Björnbergs (2000: 349ff.) an, dass die Musik ein ebenso wichtiges Element ist. Sie ist nicht beliebig austauschbar, sondern schafft Stimmung, Atmosphäre, Struktur und Assoziationen.

Die Analyse der Musik dient dem Zweck, den Sound zu beschreiben und Strukturen dieser Ebene aufzudecken. Verschiedene Kriterien, die den Rapstil der Interpretin charakterisieren, werden ebenfalls analysiert. Sinnvoll für das Herausarbeiten charakteristischer Merkmale der Musik auf der Basis einer detaillierten Analyse ist das Erstellen eines musikalischen Steckbriefs nach der Idee von Budde (1997), der Aufschluss über musikstrukturelle Elemente gibt. Um ein angemessen detailliertes Analyseergebnis zu erzielen, ist es zweckmäßig, die Auswahl und Bestimmung der Kriterien des Steckbriefes an denen von Tagg (1979: 69f.) in seiner »Check List Of Musical Considerations « zu orientieren und an das Genre Rap anzupassen. Da der voll-

9 »Rapper's Delight « war die erste kommerziell erfolgreiche Rap-Single, die jedoch nach Meinung von Experten (z.B. Toop 1992: 96ff.) weder musikalisch noch bezüglich des Rappens als charakteristisches Beispiel der damaligen Rapmusik anzusehen ist. Jedoch hat der Erfolg dieser Single das Interesse der kommerziellen Musikindustrie am Rap geweckt, wofür sie von Rapkünstlern heute noch respektiert wird. Lil' Kim zitiert den Text leicht verändert (Veränderung in eckigen Klammern). 
ständige musikalische Steckbrief (vgl. Henscher 2001) jedoch den Rahmen dieses Artikels sprengen würde, werden im Folgenden nur einige wichtige Resultate der Analyse der Musikebene des Clips dargestellt.

Insgesamt zeichnet sich die musikalische Ebene des Videoclips »No Matter What They Say« durch eine hohe Dichte einzelner Sounds und Stimmen aus sowie durch ständige Veränderungen im rhythmischen und melodischen Bereich. Dementsprechend wirkt der Gesamtsound sehr üppig und undurchsichtig, ähnlich den meisten Stücken auf Lil' Kims letztem Soloalbum The Notorious K.I.M. (2000). Die Struktur des Vokalparts verändert sich fortlaufend u.a. durch die Raps einiger Gastkünstler, durch die von P. Diddy und Lil' Kim (mit vervielfachter Stimme) ausgeführten Intensifier ${ }^{10}$ sowie durch den Wechsel von Gesang im Refrain, in dem die Stimmdichte am höchsten ist, zu Rap in Strophe und Break.

Lil' Kims Rapstil ist charakteristischer Weise überwiegend laut, aggressiv und melodisch gestaltet, sowie von, für das Genre Rap verhältnismäßig starken Tonhöhenveränderungen geprägt. Diese orientieren sich an sprachmelodischen Intonationsmustern, die teilweise stark überbetont werden. Ein rhythmisches Grund- oder ein einheitliches Akzentuierungsmuster ist nicht erkennbar. Darüber hinaus besteht ein starker Kontrast zwischen dem stilisierten Latinsound in den Strophen wie auch teilweise im Refrain zu dem Sound im zweiten Teil des Breaks. Der Latinsound wird hauptsächlich durch harmonische Mittel, die rhythmische Akzentuierung und die Ergänzung des Beats durch eine Reihe von Latin Percussion Sounds (Conga, Guiro, Shaker, Steel Drum, Cowbell, Timbale) erzeugt. Im Break wird dagegen ein in der Rapmusik klassisches rhythmisches Akzentuierungsmuster verwendet. Getragen und betont wird es durch einen elektronischen Synthesizersound, der durch seinen Klang Assoziationen an die synthetischen, orchestralen Sounds weckt, die Ende der 1980er bis Mitte der 1990er Jahre im Bereich elektronischer Popmusik häufig verwendet wurden. Diese Basisinstrumentierung und -rhythmisierung ist wohl in Ergänzung des synchron auftauchenden »Rapper's Delight «-Zitats als eine gewisse Referenz an die Anfänge des kommerziellen Raps sowie als Beleg für die Realness ${ }^{11}$ der Interpretin aufzufassen. Als mög-

10 Intensifier werden die verstärkenden Sprachfloskeln genannt, die die Textaussage an bestimmten Stellen bekräftigen sollen. Im BAE haben diese Funktion z.B. »word«, »| swear « etc.

11 Der Begriff »Realness « spielt im Rap eine große Rolle für das Erlangen von Anerkennung in der HipHop-Szene und bedeutet Echtheit oder Authentizität. Diese bezieht sich auf den sozialen und politischen Background, aus dem ein Rapper oder eine Rapperin kommt sowie auf musikalische Ästhetik und die Poesie. Sie sollte sich im Werk der Künstlerin/des Künstlers, ihrer/seiner Einstellung und Persönlichkeit widerspiegeln. 
licher Grund für die Verwendung von stereotypen Latin-Elementen kann der Trend in der Popmusik genannt werden, der seit Ende der 1990er Jahre bis heute international kommerziellen Erfolg verspricht. Die erläuterte Diskrepanz des Sounds der verschiedenen Songkonstituenten wird noch durch einen Tempowechsel, von 106 bpm (in Strophe und Refrain) auf 97 bpm (im zweiten Teil des Breaks) und wieder zurück, unterstrichen.

\section{Bildanalyse}

Der Musikvideoclip »No Matter What They Say« spielt in den Räumlichkeiten eines Schlosses. Er zeigt die Kunstfigur Lil' Kim in verschiedenen »Alltagssituationen « mit Freunden und Musikerkollegen: So probiert sie mit Freundinnen (Missy Elliott und Soulsängerin Mary J. Blige) Designermode an, begrüßt Gäste auf einer von ihr ausgerichteten Party, sitzt mit Männern zusammen und ist in choreografierten Tanzszenen mit Backgroundtänzerinnen zu sehen. Lil' Kim rappt die meiste Zeit mit Blick in die Kamera. Oft wird sie auch allein tanzend und rappend gezeigt. Der Videoclip erhält aufgrund der sich ständig ändernden filmsprachlichen Kriterien ein hohes Maß an optischer Dynamik. Einstellungsgröße, Kameraperspektive und -führung wechseln ständig.

Bei der Klassifizierung in verschiedene Strukturebenen stößt man auf ein allgemeines Problem der Rapvideoclipanalyse: Es gibt kein wirklich nützliches Modell für dieses Vorgehen in Bezug auf aktuelle Rapmusikclips. Das Modell von Altrogge und Amann (1991: 60f.), das weniger differenzierte Ansätze zur Typisierung von Clips wie den von Springsklee (1987: 127-154) erweitert, ist im Ansatz schon das fortschrittlichste. ${ }^{12}$ Die Zuordnung zu Grundtypen, wie sie bei diesem Modell vorgenommen wird, ist jedoch bei Rapvideoclips in der Regel zu vernachlässigen, da diese oft mehrere Ausprägungen der Ebenen nach Altrogge und Amann aufweisen. Dagegen ist es angebracht, alle in den Clips vorkommenden verschiedenen Darstellungs- bzw. Strukturebenen aufzuzeigen. Diese sind aber in vielen Fällen auch nicht anhand dieses Modells zu differenzieren, da sie sich häufig durch individuelle Merkmale der Präsentation der Interpretin oder des Interpreten und/oder

12 Dieses Modell teilt Videoclips in verschiedene Grundtypen nach den überwiegenden zeitlichen Anteilen des Auftretens der jeweiligen Ebene (Konzept oder Performance) im betreffenden Clip ein. Bei der Gliederung in verschiedene untergeordnete Ebenen sind u.a. das Verhältnis des Dargestellten zur genuinen musikalischen Performance und zum Musikstück sowie die Auftrittsräumlichkeiten von Bedeutung. 
durch andere bildinhaltliche oder filmsprachliche Kriterien voneinander unterscheiden, die von Altrogges und Amanns Verfahrensweise nicht berücksichtigt werden. So führt diese Methode auch beim Lil' Kim-Clip zu keinem sinnvollen Ergebnis. Dafür bietet sich indes eine Einteilung in Darstellungsebenen nach den unterschiedlichen Rollen bzw. Identitäten an, die Lil' Kim im Clip verkörpert, wie z.B. >edel gestylte Diva<, >Urban Guerilla<, >Gangsterbraut<, >blonder Vamp<, >beliebte Partygastgeberin<. Diese manifestieren sich in Kleidung, Farbgebung, Beleuchtung, Handlung sowie dem gestischen Verhalten Lil' Kims. Auf eine bestimmte Rolle lässt sich die Rapperin nicht festlegen. Doch Tanz, Bewegungen (wie z.B. Buttockshakes) und die Art der unterschiedlichen knappen Outfits unterstützen das gemeinsame Charakteristikum, das jedem Typ zugeschrieben werden kann: eine aggressive, sehr sexuelle Ausstrahlung. Nur in ihrer Identität als >Urban Guerilla gibt sich die Künstlerin nicht ganz so aufreizend. Ihre Bewegungen und ihre Kleidung im Stil der 1980er Jahre (ausgefranste, löchrige Jeans, Top mit Nietenaufschrift, Schweißarmbänder, etc.) sind hier insgesamt eher sportlich.

In ihrer äußeren Erscheinung nähert sie sich mit sehr heller Haut, dem Tragen blauer Kontaktlinsen und mit meist hellblonden, oder zumindest braunen, geglätteten Haaren auf allen Strukturebenen einem weißen Schönheitsideal an. Diese Art der Darstellung ist aber kein individuelles Phänomen. Sie ist ein Merkmal des gängigen Frauenbildes von Afro-Amerikanerinnen im aktuellen Musikvideoclip (vgl. Hoffmann 1997) und spiegelt allgemeine Tendenzen der afro-amerikanischen Kultur wieder (vgl. Arogundade 2000: 128). Allerdings ist Lil' Kims Imitation europäischer Schönheitsmerkmale in Verbindung mit ihrer oben beschriebenen sexuellen Ausstrahlung so stark übertrieben, dass ihr Auftreten insgesamt parodistische Züge erhält.

Die Demonstration des Besitzes von Statussymbolen wie Designermode, Champagner und Zigarren ist ein weiteres Merkmal, das die verschiedenen Darstellungsebenen und Identitäten Lil' Kims visuell miteinander verbindet. Hier kann im Sinne Bernd Hoffmanns von »visuellem Boasting « ${ }^{13}$ gesprochen werden, das durch die Schlosskulisse und die offenbar freundschaftlichen Verbindungen Lil' Kims zu anderen berühmten Persönlichkeiten der BlackMusic-Szene im Videoclip unterstrichen wird.

13 Analog zum sprachlichen Kommunikationsschema Boasting, als Form der verbalen Manipulation, wird beim visuellen Boasting (vgl. Anm. 5) als Form der Bildersprache des BAE das Gegenüber mit dem gleichen Ziel und den gleichen Mitteln durch die visuelle Inszenierung beeinflusst (vgl. Hoffmann 2001: 62 und Anm. 3). 
Alles in allem wird in diesem Clip das bisherige visuelle Konzept Lil' Kims weiter verfolgt und ausgebaut. Schon in ihrem Video »No Time« (1996) wurde das Boasting mit Statussymbolen wie Edelsteinen, Pelzmantel, Champagner und goldener American-Express-Karte eingesetzt. Mit blauen Kontaktlinsen und geglätteten Haaren deutete Lil' Kim auch dort schon die Merkmale eines hellhäutigen Schönheitsideals an. Im Videoclip zur Single »Crush On You« (1997) wurde auf die optische Verwandlung der Rapperin gesetzt. Die verschiedenen Darstellungsebenen sind hier durch jeweils eine Farbe gekennzeichnet, welche Outfits und Umgebung einfärbt. Auch in diesem Clip lässt sich die Interpretin, wie bei »No Matter What They Say«, auf keinen Typ bzw. keine Farbe festlegen. Hinter den verschiedenen Farben treten afro-amerikanische Merkmale zurück, wodurch eine Verdeckung des spezifischen ethnischen Hintergrunds erfolgt. Beide Clips betonen, ähnlich wie in dem hier analysierten Beispiel, die sexuellen Attribute Lil' Kims. Diese werden im auf »No Matter What They Say« folgenden Clip »How Many Licks« (2001) zusammen mit dem visuellen Boasting auf die Spitze getrieben. Lil' Kim tritt in diesem Video in den Figuren der »Candy Kim«, »Pin-up Kim« und »Nightrider Kim« auf. Die unterschiedlichen, von Lil' Kim verkörperten Typen erhalten hier Namen, die auf bestimmte Assoziationsfelder verweisen. Sie werden im Clip als lebensgroße Puppen in einer Fabrik gefertigt und zum Kauf angeboten. Alle drei Figuren haben die überzogene Zurschaustellung sexueller Reize sowie die sexuelle Animation der im Video auftretenden Männer gemeinsam. Die Sängerin prostituiert sich hier offen als künstliches Produkt zur sexuellen Befriedigung. Damit karikiert sie nicht nur ihr eigenes Image, sondern auch das von anderen afro-amerikanischen Frauen vermittelte Bild in vielen Black-Music-Videoclips.

\section{Vergleich}

Im beschriebenen Clip sind akustische, visuelle und sprachliche Ebene konzeptionell aufeinander abgestimmt. Dabei werden mehrere Textstellen im Bild indirekt oder direkt gestisch visualisiert und so die Aussagen des Textes visuell unterstützt. Doch der Hauptzweck der visuellen Präsentation liegt darin, ein Bild von Lil' Kim zu schaffen, das die übersteigerte Selbstdarstellung im Sinne des Boastings im Text untermauert. Unter Berücksichtigung der analysierten Ebenen vermittelt der Videoclip ein Bild der Rapperin als schöne, starke Frau, die sich mit Aggressivität durchsetzt. Eine Bitch im positiven Sinn, die ungemein erfolgreich und allseits begehrt ist. Zusätzlich präsentiert sie sich als fähige Rapperin mit einem direkten, teilweise 
aggressiven, »bitchy« Rapstil, der besonders in Mimik (z.B. wütend verzerrtes Gesicht) und Gestik seine visuelle Ergänzung findet. Zudem unterstützen Tanz, Gestik und Mimik die Phrasierungen des Raps durch parallele Akzentsetzung in der Körpersprache.

Die schon erläuterte Diskrepanz zwischen der Musik des zweiten Teils des Breaks und der Strophe sowie des Refrains schlägt sich auch im Bild nieder. Das Styling und die Tanzbewegungen ergänzen die Musik in beiden Fällen visuell. So verbindet z.B. im zweiten Teil des Breaks der gemeinsame Bezug auf die 1980er Jahre Tanzstil, Styling und Sound, ergänzt durch das »Rapper's Delight«-Zitat auf der Textebene. In den Strophen, sowie teilweise im Refrain und im ersten Teil des Breaks sind es Tanztechniken wie Shoulder- und Buttockshakes sowie Pelvismotionen, typische Elemente lateinamerikanischer und karibischer Tänze (vgl. Günther 1982), die mit dem stilisierten Latinsound koordiniert sind.

Die verschiedenen Rollen, die Lil' Kim visuell verkörpert, werden weder auf der Text- noch auf der Musikebene explizit angesprochen. Sie werden jedoch im Zusammenhang mit den verschiedenen Darstellungsebenen bewusst eingesetzt, um Text und Musik an entsprechender Stelle zu verknüpfen. Als Beispiel kann der Kontext des Videoclips zur zweiten Strophe angeführt werden. Textinhaltlich zeigt Lil' Kim hier den Personen, die sie und ihre Crew diffamieren, dass sie eine besonders selbstbewusste Frau ist, die sich nichts gefallen lässt und die abfällige Reden als reinen Neid auf ihre Schönheit, Begehrtheit und materiellen Reichtum auslegt. Synchron dazu erscheint die Darstellungsebene »Gangsta«, bei der die Interpretin vom männlichen Teil ihrer Crew umgeben ist, was darauf hinweist, dass sie in der Männerwelt angesehen und akzeptiert ist. Ihr knappes Outfit (Nadelstreifenkorsett und -hotpants) erhält hier durch die Ergänzung von Waffenhalfter, Pistole, Sonnenbrille und Lederhandschuhen eine gefährliche Note. In der Kulisse eines dunklen Salons mit Männern, die gelassen Zigarre rauchen, ist Lil' Kims Auftreten mit der sexy Ausführung eines Mafioso zu assoziieren. Mit dieser Darstellung kann sie den Personen, die sie diskreditieren, Respekt einflößen, Sexappeal zeigen und optisch ihre Stärke demonstrieren. Das Anfügen des bedrohlichen Elements ist jedoch nicht nur als pure Ankündigung von Gewalt oder reine Untermauerung der Darstellung als Sexobjekt zu interpretieren, denn die Handfeuerwaffe entpuppt sich im Verlauf des Videoclips als Feuerzeug und dient so der Unterstützung des parodistischen Moments der gesamten Inszenierung Lil' Kims.

Weiterhin geht in diesem Videoclip das visuelle Boasting auf der Bildebene insgesamt eine Symbiose mit dem Boasting des Textes ein, um so verstärkt ein positives Selbstbild der Künstlerin zu schaffen. Diese Ergänzung 
des Textes durch das Bild erfolgt primär in Form einer inneren Repräsentation (vgl. Hoffmann 2001: 58), d.h., Text und Bild stehen auf der Ebene des $\mathrm{BAE}$ in Bezug zueinander. Diese Beziehung zeigt sich einerseits dadurch, dass Boasting-Passagen inhaltlich kongruent visualisiert werden, ${ }^{14}$ andererseits - in Abschnitten, in denen visuelles und verbales Boasting des BAE nicht synchron und z.T. mit unterschiedlichen Symbolen ablaufen - in dem gemeinsamen Ziel: der äußerst positiven Selbstdarstellung der Interpretin, um das Gegenüber für sich einzunehmen.

Bei genauerer Betrachtung erweist sich die Beurteilung des Inszenierungskonzeptes Lil' Kims nach europäischen kulturellen Maßstäben und seine Reduktion auf eine rein willkürliche, politisch unkorrekte Überbetonung von sexuellen Attributen und Angeberei, unterlegt mit einem simplen Instrumental, als zu kurz gegriffen. Eine differenzierte Vorgehensweise sowie die Einbeziehung des kulturellen Hintergrundes sind entscheidende Faktoren zur Vermeidung einer einseitigen, kolonialisierenden Interpretation. Nur durch eine solche Verfahrensweise können die Inszenierungsstile afro-amerikanischer Rapperinnen (und auch Rapper) angemessen differenziert, analysiert und beschrieben werden. ${ }^{15}$

\section{Literatur}

Altrogge, Michael / Amann, Rolf (1991). Videoclips - Die geheimen Verführer der Jugend? Ein Gutachten zur Struktur, Nutzung und Bewertung von Heavy Metal Videoclips. Berlin: VISTAS.

Arogundade, Ben (2000). »Race Against Time.« In: Marie Claire, H. 12 (engl. Ausg.), S. 124-130.

Batschari, Aniela (1997). MTV und sein Bild der afro-amerikanischen Kultur. Eine Untersuchung unter besonderer Berücksichtigung der Sendung YO!. Alfeld/ Leine: Coppi-Verlag.

Björnberg, Alf (2000). "Structural Relationships of Music and Images in Music Video.« In: Reading Pop. Approaches to Textual Analysis in Popular Music. Hg. v. Richard Middleton. New York: Oxford University Press, S. 347-378.

Budde, Dirk (1997). Take Three Chords... Punkrock und die Entwicklung zum American Hardcore (= Schriften zur Popularmusikforschung 2). Karben: CODA.

Buß, Christoph (1998). »21st Century Blues... From Da 'Hood. Aspekte zum Thema Rap-Music.« In: Jazzforschung / Jazz Research 30. Graz: Akademische Druckund Verlagsanstalt, S. 9-99.

14 Ein Beispiel: Synchron zur Textzeile $\gg$ My rocks shine like they were dipped in Cascade« funkeln die Edelsteine eines Ringes, den Lil' Kim trägt, außergewöhnlich stark.

15 Hilfreich wäre in diesem Zusammenhang eine weitere Verbesserung bzw. Präzisierung von veralteten (Altrogge/Amann 1991) oder sehr umfangreichen Analysemodellen (Pape/Thomsen 1997). 
Dauer, Alfons M. / Hoffmann, Bernd (1987ff.). »Blues in Geschichte und Gegenwart « [15 Folgen]. In: Jazzthetik. Zeitschrift für Jazz und Anderes 1, H. 11-12 (1987); 2, Nr. 1-12 (1988); 3, Nr. 1-2 (1989).

Dufresne, David (1997). Rap Revolution. Geschichte - Gruppen - Bewegung. Aus dem Französischen von Jutta Schornstein. Mit einem Update von Günther Jacob. Zürich, Mainz: Atlantis Musikbuch-Verlag [1. Aufl. Paris: 1991].

Fernando, S. H. (1994). The new Beats. Exploring the Music, Culture, and Attitudes of Hip-Hop. New York: Anchor Books.

Glowania, Malgorzata / Heil, Andrea (1996). »Das Persönliche und das Politische. Frauen im Rap.«In: Rap. Hg. v. Wolfgang Karrer und Ingrid Kerkhoff. Berlin, Hamburg: Argument, S. 99-117.

Günther, Helmut (1982). Die Tänze und Riten der Afro-Amerikaner. Bonn: Verlag Dance Motion.

Henscher, Sonja (2001). Afro-amerikanische Rapperinnen im Videoclip. Eine vergleichende Analyse von »Doo Wop (That Thing) « (Lauryn Hill), »No matter what they say« (Lil' Kim) und »She's a Bitch« (Missy Elliott). Hamburg: unveröffentl. Magisterschrift Systematische Musikwissenschaft.

Hoffmann, Bernd (1994). »Blues. "In: Die Musik in Geschichte und Gegenwart. Sachteil Bd. 1. Hg. v. Ludwig Finscher. Kassel u.a.: Bärenreiter, Metzler (2. Aufl.), Sp. 1600-1635.

Hoffmann, Bernd (1997). »Welche Farbe hat mein Heftpflaster?« In: Musik und Unterricht. Zeitschrift für Musikpädagogik, H. 46, S. 43-51.

Hoffmann, Bernd (2001). »Anmerkungen zu poetischen und visuellen Konzepten im afro-amerikanischen Videoclip. «In: Populäre Musik im kulturwissenschaftlichen Diskurs II. Hg. v. Thomas Phleps (= Beiträge zur Popularmusikforschung 27/28). Karben: CODA, S. 53-66.

Kochman, Thomas (1972): »Towards an ethnographie of black American speech behaviour «. In: Rappin' and Stylin' Out. Communication in Urban Black America. Hg. v. Thomas Kochman. Urbana, Chicago, London: University of Illinois Press. S. 241-264.

Pape, Winfried / Thomsen, Kai (1997). »Zur Problematik der Analyse von Videoclips. «In: Step across the Border: Neue musikalische Trends - neue massenmediale Kontexte. Hg. v. Helmut Rösing (= Beiträge zur Popularmusikforschung 19/20). Karben: CODA, S. 200-226.

Rose, Tricia (1994). Black Noise. Rap Music and Culture in Contemporary America. Hanover, London: University Press of New England.

Salaam, Mtume (1996). »Rap as Art. Some Thoughts on the Aesthetics of Rap. « In: Rap. Hg. v. Wolfgang Karrer und Ingrid Kerkhoff. Berlin, Hamburg: Argument Verlag, S. 119-141.

Smitherman, Geneva (1977): Talkin and Testifyin. The Language of Black America. Boston: Houghton Mifflin Company.

Springsklee, Holger (1987). »Video-Clips - Typen und Auswirkungen.« In: film musik - video, oder Die Konkurrenz von Auge und Ohr. Hg. v. Klaus-Ernst Behne. Regensburg: Gustav Bosse Verlag, S. 127-154.

Tagg, Philip (1979). Kojak. 50 Seconds of Television Music. Towards the Analysis of Affect in Popular Music (= Studies from the Department of Musicology, Göteborg 2). Göteburg: Musikvetenskapliga Institutionen. 


\title{
Diskographie
}

Eric B. / Rakim (1987). »| Know You Got Soul.« Album: Paid In Full. Universal/ Island Records 4005.

Lil' Kim (1996). »No Time«. Album: Hardcore. eastwest/Atlantic 7567-92733-2.

Lil' Kim (1997). »Crush On You«. Album: Hardcore. eastwest/Atlantic 7567-92733-2.

Lil' Kim (2000). »No Matter What They Say«. Album: The Notorious K.I.M. eastwest/Atlantic 7567-92840-2.

Lil' Kim (2001). »How Many Licks«. Album: The Notorious K.I.M. eastwest/Atlantic 7567-92840-2.

Sugar Hill Gang (1979). »Rapper's Delight«. Album: Sugar Hill Gang. Sugarhill Records 0060.275 .

\begin{abstract}
By Western standards the concepts of the mediated images of female AfroAmerican rappers are often considered to be reactionary. But those who take this view often completely ignore the heterogeneity of individual styles and the AfroAmerican historical and cultural context. This analysis takes a closer look at the portrayal of Afro-American female rappers by taking the video clip of Lil' Kim as an example. The various layers of music, lyrics and visual material are analysed, taking the Afro-American cultural background into account. The intention is to show that a detailed approach is necessary and the specific ethnic context needs to be taken into account in order to avoid a too narrow interpretation and to describe individual styles appropriately.
\end{abstract}

